\title{
A model of the twin-cam compound bow with cam design options
}

\author{
M. Tiermas
}

Received: 6 November 2015/ Accepted: 12 February 2016/Published online: 27 February 2016

(C) The Author(s) 2016. This article is published with open access at Springerlink.com

\begin{abstract}
A mathematical model of an archery twincam compound bow is introduced. The deflection of the limb tip is based on the modified Hickman model of the traditional bow. The cams are modelled with the help of cubic splines, and the derived non-linear equations are simplified and solved numerically. The force-draw curve of a commercial twin-cam compound bow is measured and compared to the prediction of the model. As an example, the cams of the compound bow are virtually modified in order to improve the effectiveness of the bow. The model presented here can be used when adjusting the twincam compound bow and also when designing the cams.
\end{abstract}

Keywords Compound bow $\cdot$ Force-draw curve Cam design

\section{Introduction}

The archery compound bow is a bow with pulleys at the tips of the bow limbs, which offer mechanical advantage to the archer. When drawing a typical compound bow, the required force increases to the

\section{Tiermas $(\bowtie)$}

Department of Physics, University of Helsinki, Helsinki, Finland

e-mail: marko.tiermas@helsinki.fi maximum more or less rapidly, but then decreases to the local minimum value in the full draw [1]. This nonlinear behaviour can be controlled by careful design of the shape and size of the pulleys (or cams).

In the earlier studies concerning compound bow models, the pulleys at the tips of the limbs are either similar systems both consisting of two round eccentrics [2], similar systems both consisting of one round wheel and one non-round cam [3], or there is one round wheel at the other tip of the limb and the system of three non-round cams at the other [4, 5]. In the models including non-round cams, the cams are approximated as components with mixed properties of a circle and a changing lever at the same time, in order to simplify the admittedly complex system of the compound bow. However, in the viewpoint of cam design, a more detailed treatment of cams is needed.

This paper provides a more accurate solution to the problem of modelling the compound bow with nonround cams. The considerations are restricted to the bow with similar two-cam systems at the tips of the limbs. We may call this bow type as a twin-cam compound bow.

\section{Mathematical model}

Let us consider a compound bow with similar cam systems at the tips of the limbs. Let us assume that the bow is symmetric with some vertical line, which is 


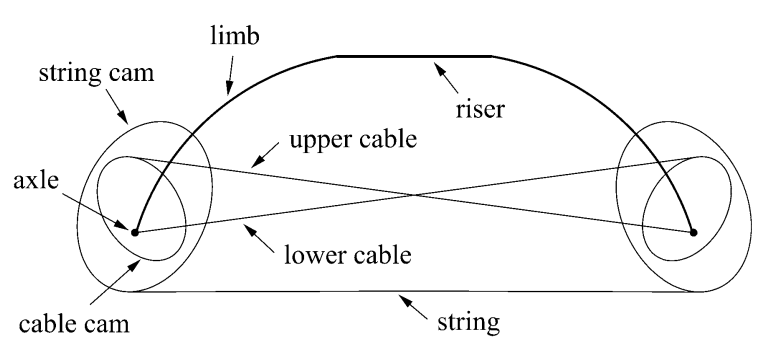

Fig. 1 The schematic picture of the twin-cam compound bow in the initial position

also the line in which the arrow moves when the bow string is drawn or released from the center of the string; the reader is noted that this is not always the case for real twin-cam bows. We further assume that the bow is in a horizontal position so that the grip and riser are above the cables and the string, and the line between the axle points of the cams is horizontal, as in Fig. 1.

The cam system at the tip of the limb consists of two cams, which are firmly attached to each other, and the system can rotate only around the axle, from which the system is connected to the tip of the limb. The string is wrapped around the string cams of both the left-hand and the right-hand limb. From one end, the upper cable is twisted around the cable cam of the left-hand limb, and the other end of this cable is connected to the axle of the right-hand cam system, as presented in Fig. 1. The lower cable is similar with respect to the right-hand cam system. In real bows there is also a cable guard, which shifts both cables slightly aside to clear the way for the arrow. However, for the sake of simplicity and symmetry we shall ignore the cable guard and assume that the cables are straight and on the same plane with the string. We shall also suppose that the cables and the string are inextensible, and that they do not slide with respect to the cam systems.

Let us also further assume that the Hickman [6] model with the modification as presented in [2] can be used to approximate the bending of the limbs, when the limb is assumed to be a rigid rod which bends only on one hinge point, which locates somewhere between the tip and the bottom of the undeflected straight limb. Due to symmetry, we may restrict our considerations to the left-hand limb and cam system of Fig. 1. The symbols used are: $\alpha \quad$ the angular coordinate of the cable cam; the angle between the ray from the axle point to the edge point of the cable cam and the polar axis (positive horizontal $\mathrm{x}$-axis with the axle point as origin) measured counter-clockwise direction in the initial position of the bow

$\beta \quad$ the angular coordinate of the string cam; the angle between the ray from the axle point to the edge point of the string cam and the polar axis (positive horizontal $\mathrm{x}$-axis with the axle point as origin) measured counter-clockwise direction in the initial position of the bow

$\gamma \quad$ the angle between the line of the cable and the horizontal line

$\delta \quad$ the angle between the horizontal line and the line that connects the axle point to the point where the straight cable touches the cam

$\varepsilon \quad$ the angle between the horizontal line and the line that connects the axle point to the point where the string touches the cam (always $>180^{\circ}$ )

$\zeta \quad$ the angle of the half-string with respect to the horizontal line

$\theta \quad$ the angle between the horizontal line and the line that connects the axle point and the supposed hinge point of the limb

$\theta_{U} \quad$ the angle between the undeflected bow limb and the horizontal line

$\varphi \quad$ the rotation angle of the cam system measured from the initial position to the counter-clockwise direction

$A$ the ratio between the the length of the supposed elastic portion of the limb with respect to the total limb length

$a$ the torque constant of the supposed elastic portion of the limb

$c$ the length of the straight cable

$D$ the draw; the distance from the midpoint of the string to the horizontal line that connects the bottoms of the limbs

$d_{c} \quad$ the lever arm of the cable tension

$d_{s} \quad$ the lever arm of the string tension

$e$ the distance between the axle points of the lefthand and the right-hand cam systems

$F \quad$ the absolute value of the force acting on the arrow

$F_{c} \quad$ the absolute value of the cable tension

$F_{s} \quad$ the absolute value of the string tension 
$g$ the distance between the bottoms of the limbs; the length of the riser

$k \quad$ the spring constant of the elastic portion of the limb

$L$ the length of the limb (from the bottom to the axle point)

$l \quad$ the vertical distance of the midpoint of the string from the line between the points where the string touches the cams of the both limbs

$m_{c} \quad$ the Cartesian slope of the tangent line to the cable cam curve at the point with polar coordinates $\left(\alpha, r_{c}\right)$ in the initial position

$m_{s}$ the Cartesian slope of the tangent line to the string cam curve at the point with polar coordinates $\left(\beta, r_{s}\right)$ in the initial position

$n_{c} \quad$ the Cartesian slope of the tangent line to the cable cam curve at the point with polar coordinates $\left(\alpha, r_{c}\right)$ after rotation angle $\varphi$ counterclockwise

$n_{s} \quad$ the Cartesian slope of the tangent line to the string cam curve at the point with polar coordinates $\left(\beta, r_{s}\right)$ after rotation angle $\varphi$ counterclockwise

$r_{c}$ the radius of the cable cam; the radial distance from the axle point to the edge point of the cable cam

$r_{s} \quad$ the radius of the string cam; the radial distance from the axle point to the edge point of the string cam

$s \quad$ the length of the straight half-string

$V \quad$ the energy stored to the limbs.

Moreover, a dot is used when referring to the derivative of the cable or string cam radius function with respect to its variable, and the subscript " 0 " is used when referring to the value of the respective variable in the initial position.

Let us choose a conventional Cartesian coordinate system and the axle point of the left-hand cam system of Fig. 1 as its origin. For the polar coordinates $(\alpha$, $\left.r_{c}(\alpha)\right)$ and $\left(\beta, r_{s}(\beta)\right)$, let us also choose a polar coordinate system with the same axle point as its pole and the positive $\mathrm{x}$-axis of the Cartesian system as its polar axis in the initial position. However, we shall fix the polar coordinates to the cam system, so if the cam system rotates with respect to the initial position the polar coordinate system rotates with it. We assume the initial values $e_{0}, L, A, k, g, \theta_{U}$ are given. If $r_{c}=r_{c}(\alpha)$ and $r_{s}=r_{s}(\beta)$ are differentiable and known in polar coordinates, the slopes to the tangent lines of these cam curves in our Cartesian system can be expressed [7] as

$m_{c}(\alpha)=\frac{r_{c}(\alpha)+r_{c}^{\prime}(\alpha) \tan \alpha}{r_{c}^{\prime}(\alpha)-r_{c}(\alpha) \tan \alpha}$

$m_{s}(\beta)=\frac{r_{s}(\beta)+r_{s}^{\prime}(\beta) \tan \beta}{r_{s}^{\prime}(\beta)-r_{s}(\beta) \tan \beta}$

Using the tangent angle addition formula, it is easy to derive the equations for the slopes of the tangent lines in our Cartesian system after the cam system has rotated counter-clockwise the angle $\varphi$,

$n_{c}(\alpha, \varphi)=\frac{\tan \varphi+m_{c}(\alpha)}{1-m_{c}(\alpha) \tan \varphi}$

$n_{s}(\beta, \varphi)=\frac{\tan \varphi+m_{s}(\beta)}{1-m_{s}(\beta) \tan \varphi}$

The following equivalences can be seen from Figs. 2, 3, 4, 5 and 7 ,

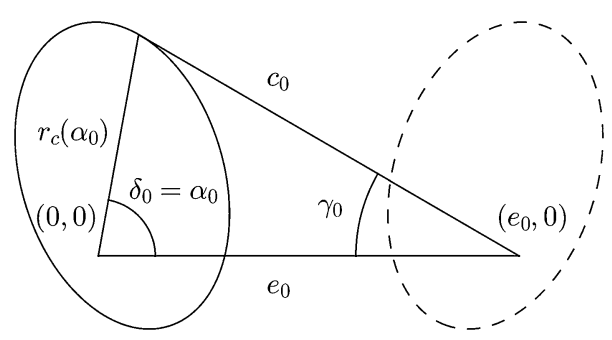

Fig. 2 The cable cams of the compound bow in the initial position with some related variables. The right-hand cam (dashed curve) is fastened on the other limb than the left-hand cam. The polar coordinate system has not rotated, so $\delta_{0}=\alpha_{0}$

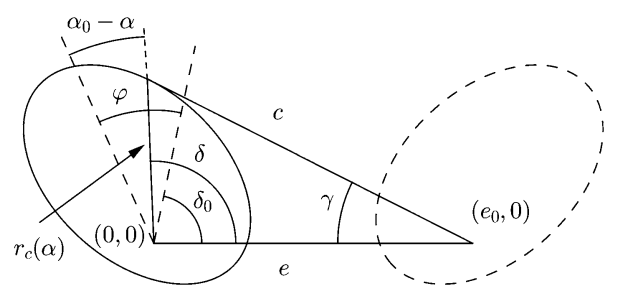

Fig. 3 The cable cams of the compound bow in the drawn position with some related variables. The right-hand cam (dashed curve) is fastened on the other limb than the left-hand cam. Compared to Fig. 2, the left-hand cam has rotated counterclockwise the angle $\varphi$ from its initial position 


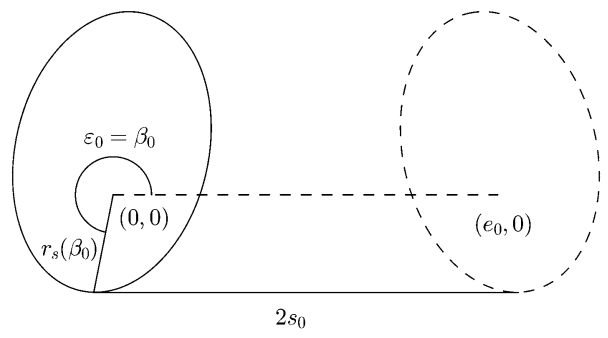

Fig. 4 The string cams of the compound bow in the initial position with some related variables. The right-hand cam (dashed curve) is fastened on the other limb than the left-hand cam. The polar coordinate system has not rotated, so $\varepsilon_{0}=\beta_{0}$

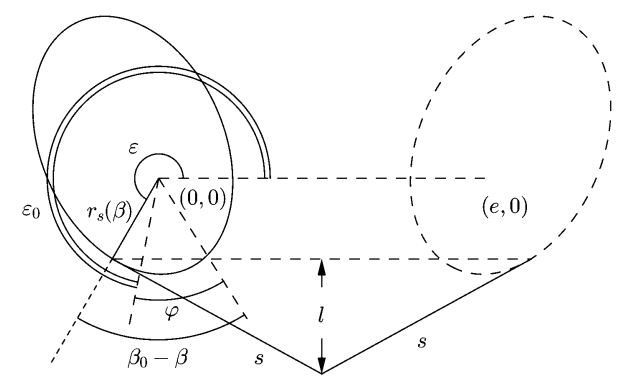

Fig. 5 The string cams of the compound bow in the drawn position with some related variables. The right-hand cam (dashed curve) is fastened on the other limb than the left-hand cam. Compared to Fig. 4, the left-hand cam has rotated counterclockwise the angle $\varphi$ from its initial position

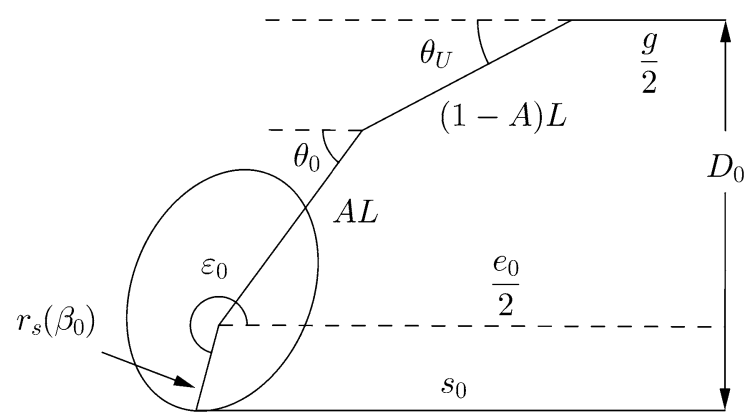

Fig. 6 The left half of the compound bow in the initial position. For clarity, the cable cam and the cables are left out

$$
\begin{aligned}
& n_{c}(\alpha, \varphi)=\tan (-\gamma) \\
& \left.n_{s}(\beta, \varphi)\right)=\tan (-\zeta) \\
& \alpha_{0}-\alpha=\delta_{0}+\varphi-\delta
\end{aligned}
$$

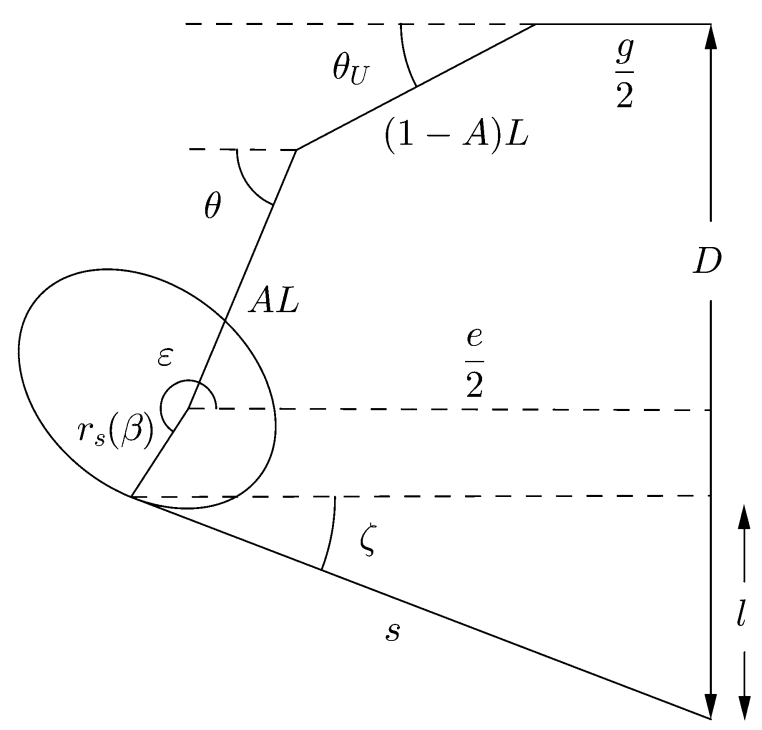

Fig. 7 The left half of the compound bow in the drawn position. For clarity, the cable cam and the cables are left out

$\beta_{0}-\beta=\varepsilon_{0}+\varphi-\varepsilon$

In the initial position (Figs. 2, 4 and 6) the polar axis of the polar coordinate system coincides with the Cartesian X-axis, hence $\alpha_{0}=\delta_{0}$ and $\beta_{0}=\varepsilon_{0}$. Then Eqs. (7) and (8) simplifies as

$\alpha=\delta-\varphi$

$\beta=\varepsilon-\varphi$

From Figs. 3 and 7 we notice that the equations for the cable and the half-string lines are

$r_{c}(\alpha) \sin \delta=n_{c}(\alpha, \varphi)\left(r_{c}(\alpha) \cos \delta-e\right)$

$l=n_{s}(\beta, \varphi)\left(r_{s}(\beta) \cos \varepsilon-\frac{e}{2}\right)$

Further, the following relations can also be seen from Figs. 3 and 7 ,

$r_{c}(\alpha)=\frac{c \sin \gamma}{\sin \delta}$

$s^{2}=l^{2}+\left(\frac{e}{2}-r_{s}(\beta) \cos \varepsilon\right)^{2}$

In the initial position the rotation angle $\varphi=0$, then $n_{c}\left(\alpha_{0}, 0\right)=m_{c}\left(\alpha_{0}\right)$ and $n_{s}\left(\beta_{0}, 0\right)=m_{s}\left(\beta_{0}\right)=0$. Remembering that $\alpha_{0}=\delta_{0}$ and $\beta_{0}=\varepsilon_{0}$, Eqs. (11) and (2) gives in the initial position 
$r_{c}\left(\alpha_{0}\right) \sin \alpha_{0}-m_{c}\left(\alpha_{0}\right)\left(r_{c}\left(\alpha_{0}\right) \cos \alpha_{0}-e_{0}\right)=0$

$r_{s}\left(\beta_{0}\right)+r_{s}^{\prime}\left(\beta_{0}\right) \tan \beta_{0}=0$

from which $\alpha_{0}$ and $\beta_{0}$ can be obtained. There is no analytical solution for these equations, so for iteration the Brent-Dekker (BD) method [8] was chosen. From Eqs. (13) and (14) we get, by using Eqs. (3), (5) and (10) in the initial position, where $\varphi=l=0$,

$r_{c}\left(\alpha_{0}\right)=\frac{c_{0} \sin \left(\arctan \left(-m_{c}\left(\alpha_{0}\right)\right)\right)}{\sin \alpha_{0}}$

$s_{0}=\frac{e_{0}}{2}-r_{s}\left(\beta_{0}\right) \cos \beta_{0}$

from which the initial values $c_{0}$ and $s_{0}$ can now be solved. The length of the straight cable and the length of the straight half-string are

$c=c_{0}-\int_{\alpha}^{\alpha_{0}} \sqrt{r_{c}^{2}(\alpha)+\left(r_{c}^{\prime}(\alpha)\right)^{2}} \mathrm{~d} \alpha$

$s=s_{0}+\int_{\beta}^{\beta_{0}} \sqrt{r_{s}^{2}(\beta)+\left(r_{s}^{\prime}(\beta)\right)^{2}} \mathrm{~d} \beta$

so $c$ can now be calculated immediately. By using Eqs. (3), (13) and (9) we may write Eq. (5) as

$\frac{\tan \varphi+m_{c}}{1-m_{c} \tan \varphi}+\tan \left[\arcsin \left(\frac{r_{c}}{c} \sin (\varphi+\alpha)\right)\right]=0$

from which $\varphi$ can be obtained with every value of $\alpha$ using BD method for example. After this is done, the unknowns $\delta, \gamma, n_{c}$ and $e$ can be calculated from Eqs. (9), (13), (3) and (11), respectively. From Eq. (14) we get, with the help of Eqs. (2), (4), (10), (12) and (20),

$$
\begin{gathered}
\left(s_{0}+\int_{\beta}^{\beta_{0}} \sqrt{r_{s}^{2}+\left(r_{s}^{\prime}\right)^{2}} \mathrm{~d} \beta\right)^{2}-\left(\frac{e}{2}-r_{s} \cos (\beta+\varphi)\right)^{2} \\
\times\left[\left(\frac{\tan \varphi\left(r_{s}^{\prime}-r_{s} \tan \beta\right)+r_{s}+r_{s}^{\prime} \tan \beta}{r_{s}^{\prime}-r_{s} \tan \beta-\tan \varphi\left(r_{s}+r_{s}^{\prime} \tan \beta\right)}\right)^{2}+1\right]=0
\end{gathered}
$$

from which $\beta$ can be obtained using BD method for example. After this, the unknowns $m_{s}, n_{s}, \zeta, \varepsilon, l$ and $s$ can be calculated from Eqs. (2), (4), (6), (10), (12) and (20). From Figs. 6 and 7 we further see that

$$
\begin{array}{r}
A L \cos \theta_{0}=\frac{e_{0}}{2}-\frac{g}{2}-(1-A) L \cos \theta_{U} \\
=A L \cos \theta+\frac{e_{0}-e}{2}
\end{array}
$$

so the angle $\theta$ and its initial value $\theta_{0}$ can be solved. The draw, as defined in this paper, is according to Fig. 7,

$D=(1-A) L \sin \theta_{U}+A L \sin \theta-r_{s}(\beta) \sin \varepsilon+s \sin \zeta$

and can now be calculated straightforwardly, likewise the distances of the axle from the cable line and from the half-string line, which are, according to Figs. 3 and 7 ,

$$
\begin{aligned}
& d_{c}=r_{c}(\alpha) \sin (\gamma+\delta) \\
& d_{s}=r_{s}(\beta) \sin (-\zeta-\varepsilon)
\end{aligned}
$$

The rest equations needed for completing the model can be concluded from paper [2],

$$
\begin{aligned}
& F_{s}=\frac{a\left(\theta-\theta_{U}\right)}{A L\left[\sin (\theta+\zeta)+\frac{2 d_{s}}{d_{c}} \sin \theta \cos \gamma\right]} \\
& F_{c}=\frac{d_{s}}{d_{c}} F_{s} \\
& F=\frac{2 d_{c} k\left(\theta-\theta_{U}\right) \sin \zeta}{d_{c} \sin (\theta+\zeta)+2 d_{s} \sin \theta \cos \gamma}
\end{aligned}
$$

where $k=\frac{a}{A L}$ is the spring constant of the elastic portion of the bow limb (N/rad).

\section{Results of model testing}

The model was first tested by using the parameters of the round-wheel compound bow measured in [2]. The string and the cable cam radius were gained by cubic spline interpolation of the polar transformations of the known eccentrics of paper [2]. The first and the second derivatives of the cubic spline are also continuous, which is an important minimum requirement for the displacement diagrams of the high-speed cam systems [9].

Using the parameter values of Table 1 in [2] the values of $\alpha_{0}$ and $\beta_{0}$ were first obtained from Eqs. (15) 
Table 1 The initial parameters of the compound bow "Smoke" used in measurements

\begin{tabular}{lllllll}
\hline Parameter & $e_{0}$ & $g$ & $\theta_{U}$ & $L$ & $A$ & $k$ \\
\hline Value & 102.0 & 42.0 & 23 & 39.2 & 0.601 & 1135 \\
Unit & $\mathrm{cm}$ & $\mathrm{cm}$ & $\circ$ & $\mathrm{cm}$ & - & $\mathrm{N} / \mathrm{rad}$ \\
\hline
\end{tabular}

and (16). The domain for the prime variable $\alpha$ was chosen to be $\alpha_{0}-260^{\circ} \leq \alpha \leq \alpha_{0}$, and 2000 evenly distributed values of $\alpha$ were selected from that domain. After this, the procedure described in Sect. 2 was executed separately with every value of $\alpha$, resulting also the respective values of $D$ and $F$.

In order to compare the force values with the same value of draw, the cubic spline function was fitted to the calculated $(D, F)$-values. Then, using the same parameter values and the same draw domain, the force-draw (FD) curve was calculated with the model of [2], using also 2000 evenly distributed knot points for the prime variable. The force differences between this model and the model presented in [2] with the same draw values were $<3 \cdot 10^{-3} \mathrm{~N}$.

Another test was made by measuring the FD curve of the twin-cam compound bow "Smoke" by Hoyt Archery. The parameters of the bow are presented in Table 1.

The values $e_{0}, g$ and $\theta_{U}$ were measured as in [2]. The limbs of the bow in question are slightly recurved, hence $A$ and $L$ were estimated together from the measured limb tip data using Levenberg-Marquardt algorithm [10] for curve fitting as described in [2].

The FD curve of the compound bow was measured with the arrangements explained in [2]. The value of the constant $k$ was set only after the measurements of the FD curve and the string and cable cams. The constant $k$ was chosen so that the model fits to the measurements as well as possible in the sense of least squares.

In order to find the cable and the string cam radius functions $r_{c}(\alpha)$ and $r_{s}(\beta)$, the cams were photographed, and the radius of the string and the cable cam with different cam angles were measured from the enlarged photo. The grooves for the cable and the string were also taken into account as in [2]. The cable and the string cam radius functions were then gained by cubic spline interpolation of the angle-radius data of the cams. It turned out that the data needed additional processing. We shall discuss about this later on. The shapes of the cams, which can be also

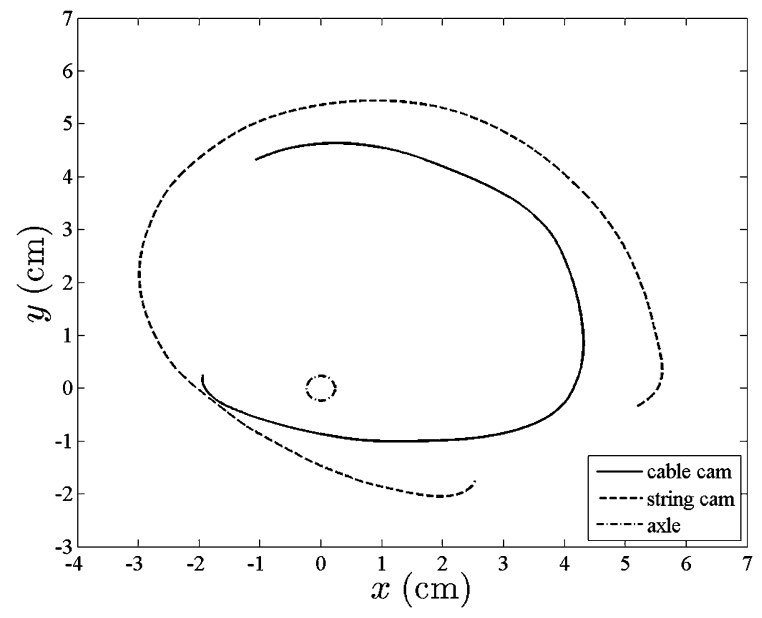

Fig. 8 The string and the cable cam in the initial position. The diameter of the axle is $4.75 \mathrm{~mm}$

concluded from the before mentioned cam radius functions using Cartesian conversion, are presented in Fig. 8.

The cams in Fig. 8 are in the same initial position as the left-hand cams in Figs. 1, 2, 4 and the cam in Fig. 6. Using the cable and the string cam radius functions and the bow parameters of Table 1, the FD curve of the model was calculated as before using $2000 \mathrm{knot}$ points for the prime variable $\alpha$ in the domain of $\alpha_{0}-235^{\circ} \leq \alpha \leq \alpha_{0}$, where $\alpha_{0}$ was first obtained from Eq. (15). The calculated FD curve of the model and the measured FD data are presented in Fig. 9.

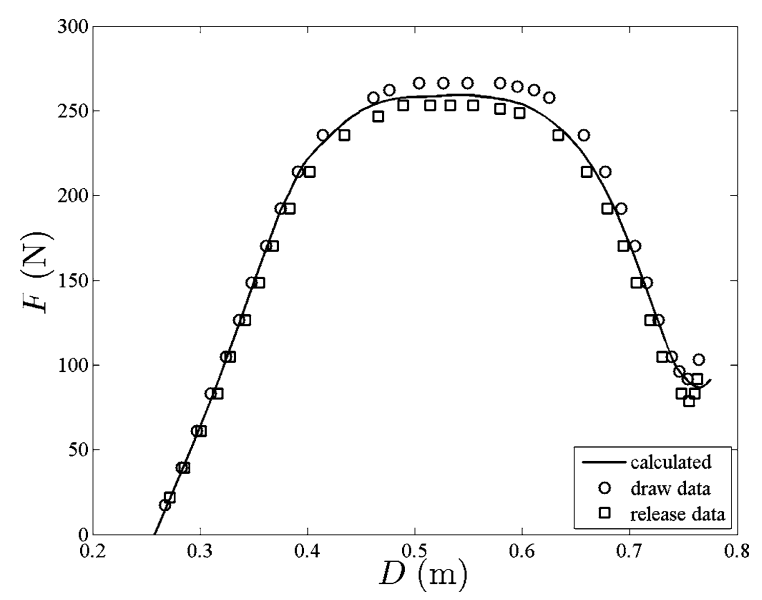

Fig. 9 The calculated force-draw curve and the measurement data of the compound bow "Smoke" 
We notice from Fig. 9 that the model match on the FD data is good. We also see that while drawing the bow, the force values are systematically bigger when compared to the values of relaxing the bow. Evidently this is caused by the friction of the wheels and the hysteresis of the limbs, string and cables. In the full draw the calculated curve is a bit aside the measured FD data. The reason for this is probable the elongation of the string and the cables, and the small measurement errors of the cams.

The area between the FD curve of the compound bow and draw-axis defines also the energy stored to the bow limbs,

$$
V(D)=\int_{D_{0}}^{D} F(D) \mathrm{d} D
$$

We may also write the potential energy stored to the limbs as in [2],

$$
V(\theta)=A L k\left(\theta^{2}-\theta_{0}^{2}+2 \theta_{0} \theta_{U}-2 \theta \theta_{U}\right)
$$

which can be used for checking the computations. With the parameter values presented in Table 1 the cubic spline function which was fitted to the $(D, F)$ values of the model was integrated numerically. Using the draw from the initial position to the draw of $D=0.764 \mathrm{~m}$ and 2000 knot points, the differences between the calculations based on Eqs. (30) and (31) were $<0.1 \%$.

\section{Other results}

With the model introduced, an individual twin-cam compound bow can now be virtually adjusted by the user. After the parameters of Table 1 and the parameters concerning the cams are measured, it is possible virtually for example to rotate the cams or change the initial limb angle and check the effects on the FD curve and on the string or cable forces. On the following we shall consider the interesting possibility of cam design.

Let us take the rather "aggressive" cam measured before as our starting point and try to modify it such a way that the FD curve is even more energetic, with the following restrictions:

1. The original full draw may not change significantly,
2. The maximum force of the FD curve may not change significantly.

The shape of the FD curve which satisfies these restrictions and can not be made more energetic is a perfect rectangle. In this sense our example bow is not quite optimal, for according to Fig. 9 there seems to be some room in both the rising and the descending part of the curve.

When manipulating the cam data by trial and error it was found that even one slightly divergent data point in the cam data may cause serious computational problems. This is due to the fact that both the cable and the string cam must be convex everywhere, otherwise the contact point of the cable (or string) and the cam will "jump" on the cams in impractical way. The respective condition equations for the cable and string cam radius functions are

$m_{c}^{\prime}(\alpha)>0$

$m_{s}^{\prime}(\beta)>0$

which after differentiating Eqs. (1) and (2) and simplifying can be expressed as

$$
\begin{aligned}
& r_{c}^{2}+2\left(r_{c}^{\prime}\right)^{2}-r_{c} r_{c}^{\prime \prime}>0 \\
& r_{s}^{2}+2\left(r_{s}^{\prime}\right)^{2}-r_{s} r_{s}^{\prime \prime}>0
\end{aligned}
$$

It was not a simple task to fulfil these condition equations everywhere on the domain of the cam radius functions. This was also the reason why the original cable and string cam angle-radius data measured in Sect. 3 had to be smoothened by measuring and inserting some additional data points and leaving out some troublesome data points.

Finally, by trial and error the more "aggressive" cable and string cam radius functions which satisfy the restrictions 1 and 2 and also Eqs. (34) and (35) were found. The respective modified cable and string cams are presented in Fig. 10, where the cams are again in the initial position as in Fig. 8.

The calculated FD curves with both the original and the modified cams are presented in Fig. 11. The bow parameters of Table 1 were used in the calculations of both curves. From Fig. 11 we notice that the FD curve with the modified cams is more energetic mainly for the more pronounced front part. Note also that with the modified cams, the initial draw is reduced. The 


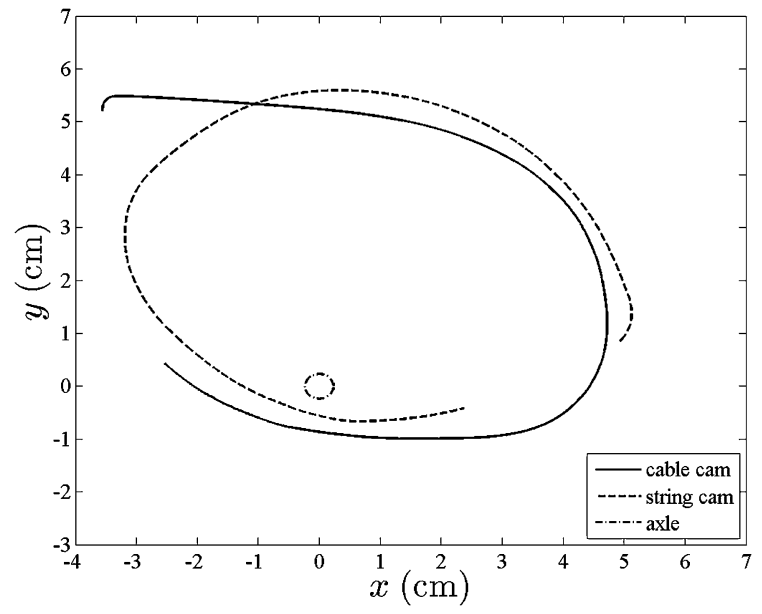

Fig. 10 The modified string and the cable cam in the initial position. The diameter of the axle is $4.75 \mathrm{~mm}$

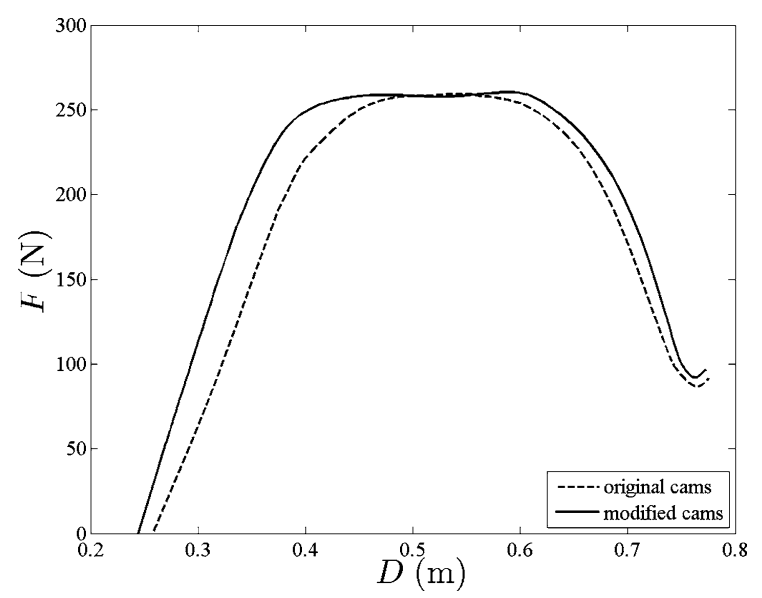

Fig. 11 The calculated force-draw curves of the compound bow "Smoke" with the original and the modified cams

calculated energy from the initial to the draw $D=0.764 \mathrm{~m}$ is $96.5 \mathrm{~J}$ for the bow with original cams and $106.5 \mathrm{~J}$ for the bow with modified cams, so the increment of energy is $10 \%$. Moreover, this was only one example of modifying the cams while seeking the maximum energy of the bow with the restrictions 1 and 2 mentioned before. Yet, with real cams it must always be checked whether the minimum radius of the cable and the string cams are sufficient in the viewpoint of material strength, for the axle has dimensions as well.

\section{Conclusion}

A mathematical model of an archery compound bow with non-round cams is introduced. The shapes of the string and the cable cams were modelled with the help of cubic splines. The non-linear equations of the model were simplified and solved numerically. The consistency and the validity of the model were checked in several ways, and the model was found to be accurate.

The path of the limb tip can be approximated with the modified Hickman model also in case when the limbs are slightly recurved. However, if the profile of the undeflected limb differs from straight rod, it is reasonable to estimate parameter $L$ (the value of "straight" limb length) together with constant $A$ by curve fitting.

It turned out that the model is particularly sensitive to the cam radius functions $r_{c}$ and $r_{s}$. When modelling the cams, it must be checked that Eqs. (34) and (35) are valid everywhere on the domain of the cam radius functions.

With the help of the model, the cams of an individual bow can be virtually modified for example to get the FD curve of the bow more effective. Depending on the desired shape of the FD curve this may be a hard job in practice.

The model presented here offers interesting possibilities when adjusting the twin-cam compound bow and when designing the cams. The reader is still reminded that the model is static only.

Acknowledgments This research was financially supported by Byro Energiatekniikka Oy. I want also to express my gratitude to Juha Kylmä and Tuomas Välimäki for the technical support and assistance with the measurements.

Open Access This article is distributed under the terms of the Creative Commons Attribution 4.0 International License (http:// creativecommons.org/licenses/by/4.0/), which permits unrestricted use, distribution, and reproduction in any medium, provided you give appropriate credit to the original author(s) and the source, provide a link to the Creative Commons license, and indicate if changes were made.

\section{References}

1. Aronson RB (1977) The compound bow: ugly but effective. Mach Des 10(25):38-40 
2. Tiermas M (2015) An advanced model of the round-wheel compound bow. Meccanica. doi:10.1007/s11012-0150262-5

3. Zanevskyy IP (2012) Compound archery bow asymmetry in the vertical plane. Sports Eng 15:167-175

4. Park JL (2009) A compound archery bow dynamic model, suggesting modifications to improve accuracy. Proc Inst Mech Eng Part P J Sports Eng Technol 223:139-150

5. Park JL (2009) Compound archery bow nocking point locus in the vertical plane. Proc Inst Mech Eng Part P J Sports Eng Technol 224:141-154

6. Hickman CN (1937) The dynamics of a bow and arrow. J Appl Phys 8:404-409
7. Courant R (1966) Differential and integral calculus, vol I. Blackie \& Son Ltd, London

8. Brent RP (1973) Algorithms for minimization without derivatives. Prentice Hall, Englewood Cliffs

9. Shigley JE, Uicker JJ (1981) Theory of machines and mechanisms. McGraw-Hill, New York

10. Marquardt D (1963) An algorithm for least-squares estimation of nonlinear parameters. SIAM J Appl Math 11(2):431-441 